\title{
BMJ Open Quality Improving delivery of low tidal volume ventilation in 10 ICUs
}

\author{
Chenell Donadee (iD , ${ }^{1}$ Mark Cohen-Melamed, ${ }^{2}$ Edgar Delgado, ${ }^{2}$ Scott R Gunn (D) ${ }^{1}$
}

To cite: Donadee C, CohenMelamed M, Delgado E, et al. Improving delivery of low tidal volume ventilation in 10 ICUs. BMJ Open Quality 2022;11:e01343. doi:10.1136/ bmjoq-2021-001343

Received 7 January 2021 Accepted 20 January 2022
Check for updates

(C) Author(s) (or their employer(s)) 2022. Re-use permitted under CC BY-NC. No commercial re-use. See rights and permissions. Published by BMJ.

${ }^{1}$ Department of Critical Care Medicine, University of Pittsburgh, Pittsburgh, Pennsylvania, USA

${ }^{2}$ Respiratory Care Department, University of Pittsburgh Medical Center, Pittsburgh, Pennsylvania, USA

Correspondence to Dr Chenell Donadee; donadeecl@upmc.edu

\section{ABSTRACT}

Low tidal volume ventilation (LTVV) is standard of care for mechanically ventilated patients with acute respiratory distress syndrome and has been shown to improve outcomes in the general mechanically ventilated population. Despite these improved outcomes, in clinical practice the LTVV standard of care is often not met. We aimed to increase compliance with LTVV in mechanically ventilated patients in 10 intensive care units at 3 hospitals within the University of Pittsburgh School of Medicine Department of Critical Care Medicine. Four Plan-DoStudy-Act (PDSA) cycles were implemented to improve compliance with LTVV. Initial compliance rates of $40.6 \%$ $60.1 \%$ improved to $91 \%-96 \%$ by the end of the fourth PDSA cycle. The most impactful step in the intervention was providing education and giving responsibility of selecting the tidal volume to the respiratory therapist. The overall intervention resulted in improved compliance with LTV that has been sustained for multiple years after our active PDSA cycles.

\section{PROBLEN}

Low tidal volume ventilation (LTVV) reduces mortality in patients with acute respiratory distress syndrome (ARDS), having reduced mortality from $39.8 \%$ to $31 \%$ in a landmark clinical trial. ${ }^{1}$ Since publication of this trial in 2000, other studies suggest possible improvement in outcomes using LTVV for patients without ARDS. ${ }^{23}$ Accordingly, LTVV is recommended in clinical practice guidelines. ${ }^{4-6} \mathrm{Yet}$, despite its acceptance as a standard of care treatment for patients with ARDS, a recent study showed that LTVV was achieved in less than two-thirds of patients with ARDS. ${ }^{7}$ An observational study of mechanically ventilated patients with and without ARDS in six intensive care units (ICUs) found that $40 \%$ of patents did not receive LTVV at some point in their hospital course. ${ }^{8}$

The Adult Division of the University of Pittsburgh School of Medicine Department of Critical Care Medicine operates 10 ICUs at 3 hospitals within the University of Pittsburgh Medical Center (UPMC) healthcare system, located in western Pennsylvania. These ICUs have a total of 167 beds and are responsible for approximately 44846 patient admissions per year. They are a mix of general and specialty ICUs including obstetrics, burn, trauma, neurovascular, cardiothoracic surgery and transplant ICUs. Six of the ICUs are located at UPMC Presbyterian Hospital. This hospital also has a cardiac ICU and medical ICU (MICU) that are not staffed by the Department of Critical Care Medicine and were not included in the intervention, although their data were also collected to serve as control groups. All ICU beds at the two other hospitals are run by the Department of Critical Care Medicine and were included in this study (table 1).

The initial opinion of physicians within the Department of Critical Care Medicine was that compliance with LTVV was probably no better than in the published data cited, but prior to this quality improvement (QI) initiative there was no way to obtain baseline data or monitor compliance through the electronic medical record (EMR). To address the perceived gap in clinical practice, the aim of this QI initiative was to improve compliance with LTVV, defined for this project as $\leq 8 \mathrm{~mL} / \mathrm{kg}$ predicted body weight (PBW), in all mechanically ventilated patients in all ICUs that operated under the Department of Critical Care Medicine. This quality initiative was supported by departmental leadership, nursing leadership, the medical directors of each ICU and the directors of the respiratory care departments at each hospital involved in the initiative.

\section{BACKGROUND}

ARDS is a pulmonary process defined by bilateral opacities not fully explained by effusions. This pulmonary process (1) occurs within 1 week of a known clinical insult or new worsening respiratory symptoms, (2) is not fully explained by cardiac failure or fluid overload and (3) results in $\mathrm{a} \mathrm{PaO}_{2} / \mathrm{FiO}_{2}$ ratio $<300 \mathrm{~mm}$ Hg. ${ }^{9}$ Risk factors for the development of ARDS include sepsis, trauma, pneumonia, aspiration, inhalational injury and blood transfusion, among others. ${ }^{10}$ It is associated with an inflammatory reaction to alveolar injury, and its histological correlate is widely considered to be diffuse alveolar damage. ${ }^{10}$ 


\begin{tabular}{|c|c|c|c|}
\hline Hospital & ICU & Number of beds & Annual admissions \\
\hline \multicolumn{4}{|c|}{ Intervention group } \\
\hline \multirow[t]{6}{*}{ Presbyterian } & Transplant ICU & 12 & 3447 \\
\hline & Trauma ICU & 22 & 7171 \\
\hline & Surgical ICU & 8 & 580 \\
\hline & Cardiothoracic ICU & 28 & 5418 \\
\hline & Neurovascular ICU & 20 & 6900 \\
\hline & Neurotrauma ICU & 10 & 3355 \\
\hline \multirow[t]{3}{*}{ Mercy } & MICU & 20 & 5431 \\
\hline & Cardiovascular ICU & 24 & 6173 \\
\hline & Trauma/Burn ICU & 9 & 2564 \\
\hline Magee & ObGyn ICU & 14 & 3807 \\
\hline \multicolumn{4}{|c|}{ Control group } \\
\hline \multirow[t]{2}{*}{ Presbyterian } & CCU & 10 & 3257 \\
\hline & MICU & 24 & 8225 \\
\hline
\end{tabular}

CCU, cardiac care unit; ICU, intensive care unit; MICU, medical ICU.

ARDS is associated with a $27 \%-45 \%$ mortality. ${ }^{9}$ ARDS is estimated to affect 190000 patients in the USA annually. ${ }^{11}$ Internationally, ARDS is present in approximately $10 \%$ of all ICU admissions and $23 \%$ of mechanically ventilated patients. ${ }^{7}$ Of patients that survive the acute illness, many have persistent physical, psychological and quality of life impairments that persist for at least 5 years after the acute event. $^{12}$

Despite the high prevalence of ARDS in ICUs and the high associated morbidity and mortality, it has been shown that ARDS is recognised in only $34 \%$ of cases by the treating physicians. ${ }^{7}$ While a handful of interventions have been shown to improve oxygenation or decrease mechanical ventilator days, only LTVV and prone positioning have been shown to improve mortality in this population. ${ }^{113}$ Of these therapies, LTVV has the greatest amount of supporting evidence and is recommended as standard of care in other trials of ARDS therapy. 1314

LTVV may also benefit mechanically ventilated patients without ARDS. Patients who receive LTVV after undergoing major surgery have fewer respiratory complications such as development of atelectasis and pneumonia, have a lower rate of reintubation and have a decreased hospital stay as compared with those who receive larger tidal volumes. ${ }^{2}$ LTVV use in patients without ARDS is also associated with a decrease in the risk of development of lung injury, pulmonary infection and risk of developing ARDS. Mortality benefit in patients without ARDS has been noted in some meta-analyses but not in others. ${ }^{15} 16$

The high prevalence but low recognition of ARDS suggests a delay in proper treatment, especially with LTVV. A retrospective analysis of four US hospitals showed that only $54 \%$ of patients with ARDS received a tidal volume $<8 \mathrm{~mL} / \mathrm{kg}$ PBW at some point during their illness, while an international observational study demonstrated only $65 \%$ compliance with LTVV. ${ }^{77}$ We had no reason to suspect our recognition of ARDS or compliance with LTVV was any better. We proposed a QI project that enacted operational and system changes in order to improve compliance with LTVV in mechanically ventilated patients.

\section{MEASUREIMENT}

The active phase of this QI initiative spanned 2 years. We collected baseline data retrospectively in 2015 using an electronic record-based report built as one of our PDSA cycles. We generated baseline tidal volume data by averaging all charted tidal volumes for mechanically ventilated patients during each day of ventilation and calculated an average tidal volume. Using this average tidal volume, we calculated compliance with LTVV $(\leq 8 \mathrm{~mL} / \mathrm{kg}$ PBW) compared with the following predicative formulas: for male patients, PBW $(\mathrm{kg})=50+2.3$ (height (in) -60$)$ and for female patients, PBW $(\mathrm{kg})=45.5+2.3$ (height (in)60). ${ }^{1}$ We performed all calculations from data that was already in the EMR so there was no additional data collection for frontline medical staff. Compliance was defined as the percentage of days per month with an average tidal volume $\leq 8 \mathrm{~mL} / \mathrm{kg}$ PBW divided by the total number of days of mechanical ventilation in that month by ICU over time.

It is important to note that in our initiative LTVV was applied to all mechanically ventilated patients rather than just those with formally diagnosed ARDS.

We generated statistical process control (SPC) charts using QI Macros in Microsoft Excel. ${ }^{18}$ We produced SPC charts by hospital and ICU. At UPMC Presbyterian Hospital, the MICU and cardiac ICU did not participate in the QI project and thus their data were not included in figure 1. We constructed SPC charts for those ICUs separately (figures 2 and 3 ). 

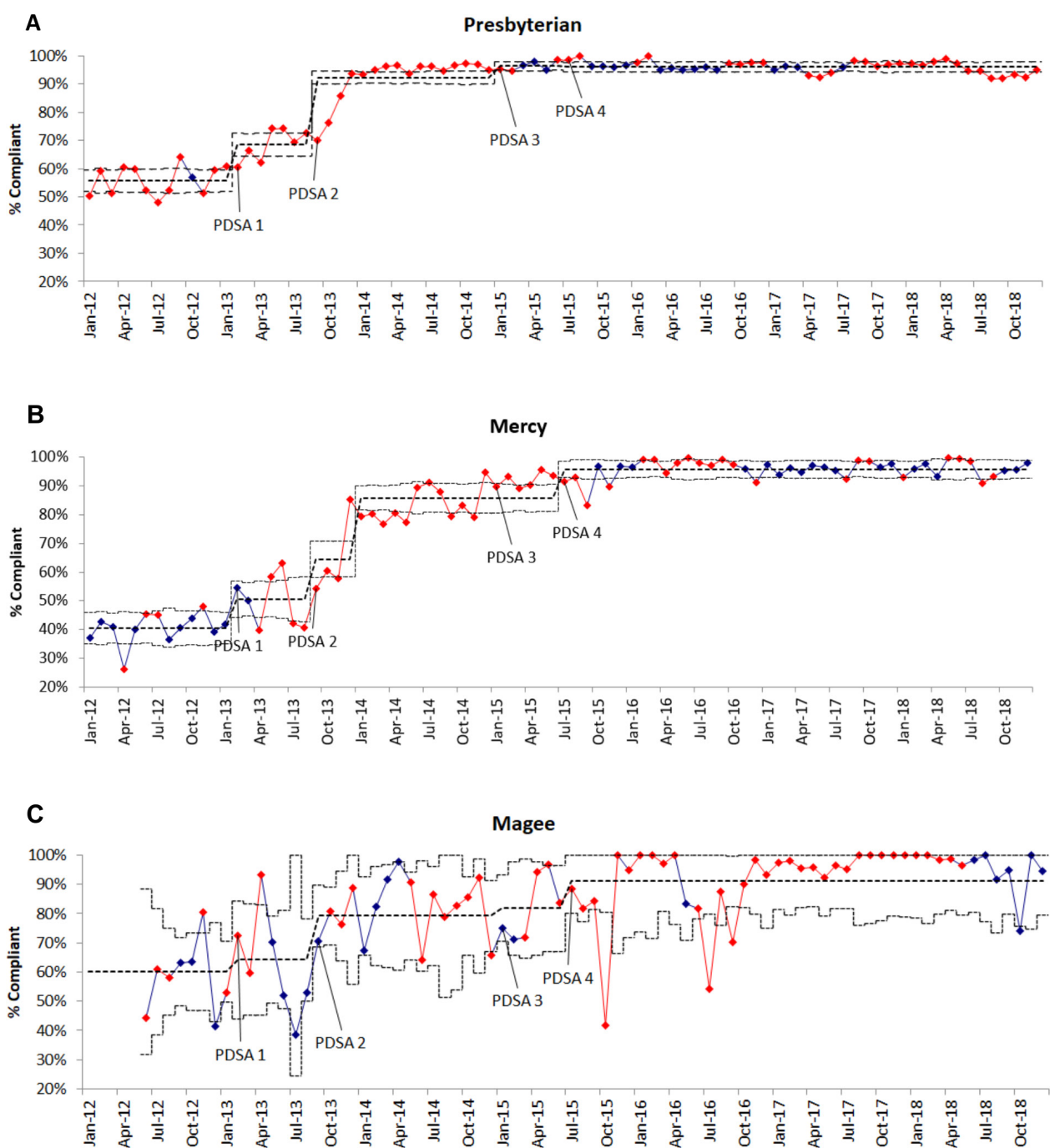

Figure 1 Changes in compliance with low tidal volume ventilation (LTVV). Statistical process control charts illustrating changes in LTVV over time in intensive care units (ICUs) at the three hospitals participating in the quality improvement project. (A) Changes in compliance with LTVV at Presbyterian Hospital. (B) Changes in compliance with LTVV at Mercy Hospital and (C) Changes in compliance with LTVV at Magee Hospital. Per cent of mechanically ventilated ICU patient days with LTVV compliance is charted on the $y$-axis and time is charted on the $x$-axis. Plan-Do-Study-Act (PDSA) cycles 1-4 are noted. Horizontal light dashed lines represent the upper and lower confidence limits. Dark dashed lines represent the centre line. Red diamonds represent unstable trends. Blue diamonds represent stable trends.

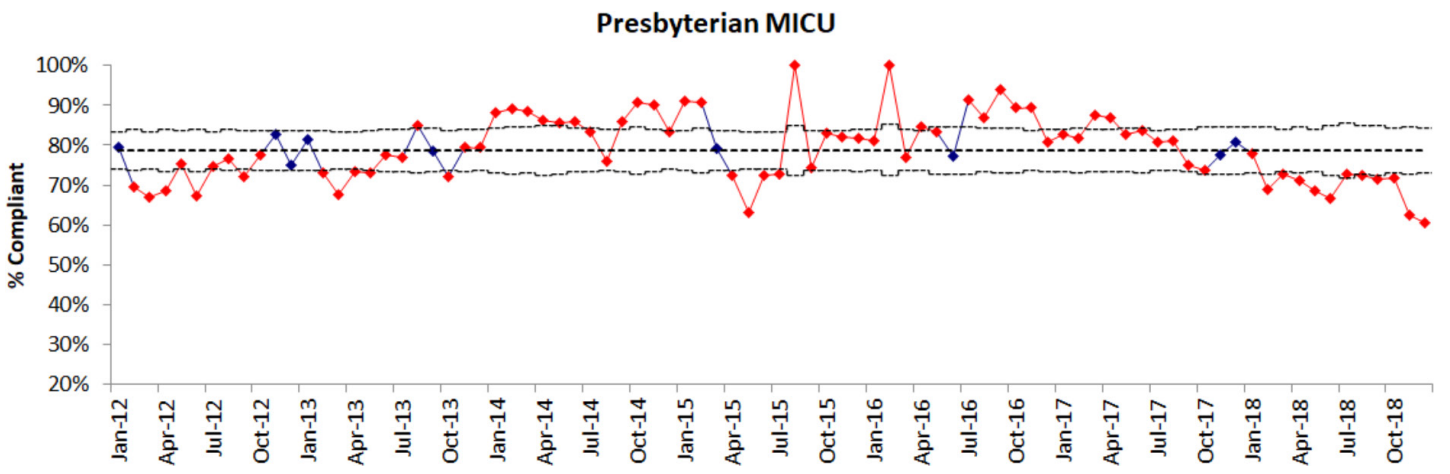

Figure 2 Compliance with low tidal volume ventilation (LTVV) in the Presbyterian medical intensive care unit (MICU). Statistical process control charts illustrating compliance with LTVV over time in the Presbyterian MICU, which did not actively participate in the quality improvement project. Per cent of mechanically ventilated ICU patient days with LTVV compliance is charted on the $y$-axis and time is charted on the $x$-axis. Horizontal light dashed lines represent the upper and lower confidence limits. Dark dashed lines represent the centre line. Red diamonds represent unstable trends. Blue diamonds represent stable trends. 


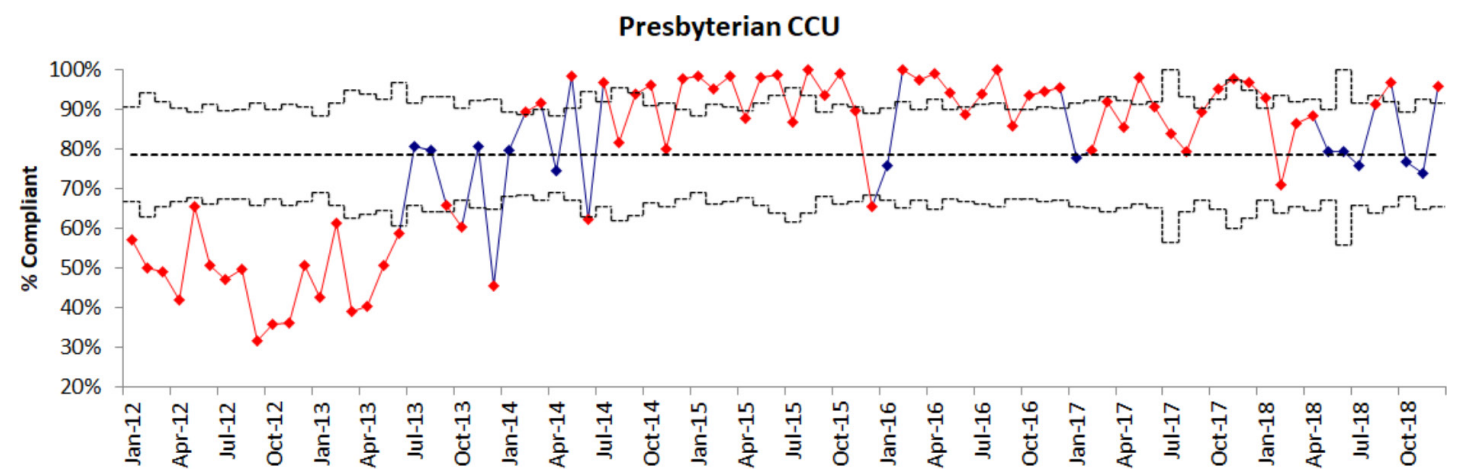

Figure 3 Compliance with low tidal volume ventilation (LTVV) in the Presbyterian coronary care unit (CCU). Statistical process control charts illustrating changes in LTVV over time in the Presbyterian CCU, which did not actively participate in the quality improvement project. Per cent of mechanically ventilated ICU patient days with LTVV compliance is charted on the y-axis and time is charted on the $x$-axis. Horizontal light dashed lines represent the upper and lower confidence limits. Dark dashed lines represent the centre line. Red diamonds represent unstable trends. Blue diamonds represent stable trends.

\section{DESIGN}

The QI strategic plan involved multiple approaches, including development of an EMR report, initiatives to decrease tidal volumes, education and tying outcomes of the project to physician compensation. Many of these steps were deployed in overlapping or simultaneous time periods and are presented in our annotated SPC charts (figure 1).

\section{STRATEGY}

First, we built consensus among the attending physicians in our department and collaborated with the directors of the respiratory care departments at all three hospitals. We undertook a number of overlapping interventions in the form of Plan-Do-Study-Act (PDSA) cycles over a period of 2 years as outlined in the following PDSA cycles. We identified project champions in each ICU to promote and support the changes. These champions were leaders in the Department of Critical Care Medicine, the chief of service of critical care at each hospital, physician medical directors and nursing unit directors in each participating ICU, as well as directors of the respiratory care departments at each hospital.

\section{PDSA cycle 1: setting to average tidal volumes for sex (February 2013-August 2013)}

Our goals for PDSA cycle 1 were twofold: (1) to use a simplistic, standardised initial tidal volume that would be easy to implement to begin improving compliance with LTVV and (2) to get physicians, respiratory therapists and nursing staff acclimatised to lower tidal volumes. We used the CDC 2012 Anthropometric Reference Data for the average heights for adult men and women in the USA and calculated the average tidal volumes for adult men and women. The average height of men was 70 inches, which translates to a $438 \mathrm{~mL}$ tidal volume at $6 \mathrm{~mL} / \mathrm{kg}$ PBW, which we rounded to a $440 \mathrm{~mL}$ tidal volume for ease of use. The average height of women was 63.8 inches, which results in a $325 \mathrm{~mL}$ tidal volume at $6 \mathrm{~mL} / \mathrm{kg}$ PBW, which we rounded to
$330 \mathrm{~mL}$ for ease of use. ${ }^{19}$ Our aim was that all mechanically ventilated men would initially be placed on a tidal volume of $440 \mathrm{~mL}$ and all women would be placed on a tidal volume of $330 \mathrm{~mL}$.

While this intervention did improve overall compliance with LTVV, it was not likely to improve compliance with LTVV for individual patients whose height was much above or below the average heights for each sex. Feedback and analysis of this cycle informed the next PDSA cycle, which aimed to improve compliance with LTVV ventilation in all mechanically ventilated patients regardless of each individual patient's height.

PDSA cycle 2: education, respiratory therapist-driven tidal volumes and implementation (September 2013-January 2015)

Our aim for PDSA cycle 2 was to implement individualised LTVV for each mechanically ventilated ICU patient based on their height and sex. We implemented this practice change using multiple change management methods, including (1) engaging and achieving buy-in from key stakeholders in each ICU, (2) creating clear roles and responsibilities, (3) developing and using tools to aid the initiative and (4) providing educational sessions.

Before beginning PDSA cycle 2, we conducted multiple individual meetings with key stakeholders from nursing, respiratory and physician groups to create buy-in. In addition, we defined clear roles for each group. Based on input from our frontline providers, we decided that nursing staff would be responsible for recording a correct height for each patient. Importantly, we empowered our respiratory staff with the responsibility and autonomy to select the initial tidal volume. The treating physician could subsequently request to have the tidal volume changed if they felt it was medically necessary. We also set an expectation that when the alternative tidal volume was no longer necessary, the default tidal volume should return to $\leq 8 \mathrm{~mL} / \mathrm{kg}$ PBW. A process map of the baseline process and new process that was implemented is laid out in figure 4. 
Baseline Process
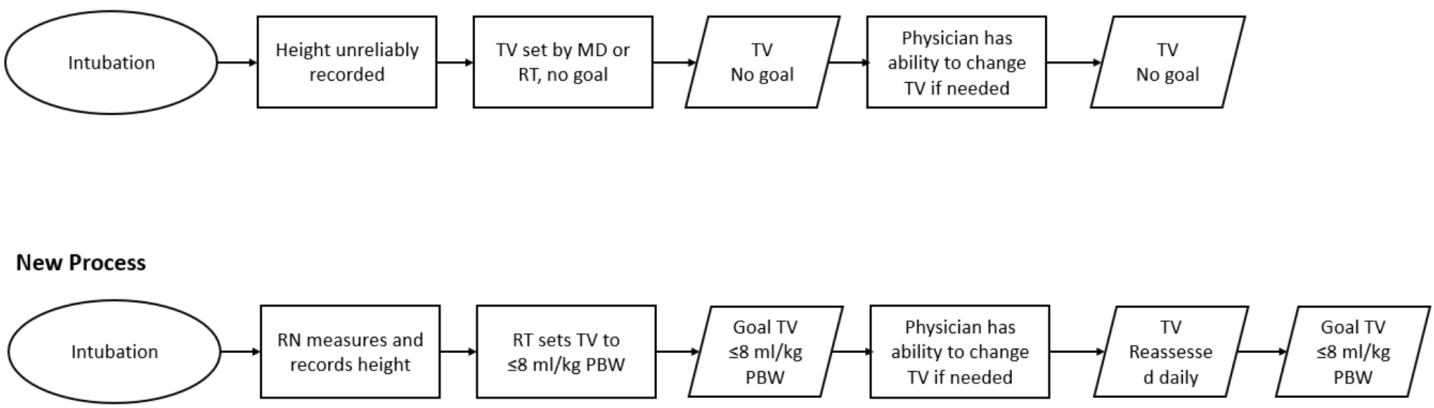

Figure 4 Process map of baseline process and new process implemented for setting tidal volumes. The baseline process is the process that existed prior to the quality improvement project. The new process was implemented as part of Plan-Do-StudyAct (PDSA) cycle 2. PBW, predicted body weight.

Our discussions with key leaders about these roles and responsibilities led to the development of multiple tools to aid frontline staff with the project. We recognised that not all nursing staff had easy access to measuring tapes to measure patient height, so we made measuring tapes readily available in the patient rooms as part of each room's standard supplies. At the request of respiratory care leadership, we created tidal volume pocket cards and distributed them to respiratory therapists and physicians for easy reference. Many ICUs also chose to write the patient's height and goal $6 \mathrm{~mL} / \mathrm{kg}$ PBW tidal volume on white boards in the patient room or in bedside respiratory therapy paper charting so it was always readily available for staff to reference. We added EMR functionality so that set tidal volumes were also displayed in $\mathrm{mL} /$ $\mathrm{kg}$ PBW and were highlighted in red if the tidal volume was $>8 \mathrm{~mL} / \mathrm{kg}$ PBW.

We developed customised educational content for each group of staff involved with the project and tailored it to their responsibilities. In our educational efforts, we emphasised that LTVV reduces morbidity and mortality. We subsequently realised that there was variation in how nursing staff was obtaining heights and these heights were being inconsistently documented, so we provided ongoing audit, feedback and education at nurse staff meetings. Respiratory staff education, provided at staff meetings, emphasised how to determine a tidal volume $\leq 8 \mathrm{~mL} / \mathrm{kg}$ PBW for each patient.

We provided education for physicians on the importance of LTVV and built awareness of this QI project at faculty meetings as well as fellow and resident lectures. We involved residents and fellows in teaching educational sessions to their peers. For resident and fellow education, we developed supplemental materials that explained the evidence behind the initiative. We provided this information to residents and other trainees who were rotating through our ICUs and may not have been familiar with evidence.
PDSA cycle 3: development of an electronic medical record report (January 2015-July 2015)

Our objective for PDSA cycle 3 was to fine tune our previously developed EMR report so we could provide accurate audit and feedback. UPMC uses Cerner PowerChart for all inpatient activities. Using this report, the previous month's results were reported monthly to the physician ICU medical directors of each ICU. We updated the report so the physician and respiratory therapist could more readily determine whether the patient was meeting the LTVV standard of care. The updated report excluded all instances in which a patient was recorded as being on pressure support mode or a spontaneous breathing trial, since the tidal volumes vary by patient effort and higher tidal volumes on these weaning modes would not be a reason to stop a breathing trial. In addition, we found that the report could not accommodate patients who had multiple heights charted in their EMR, because an error would result. We solved this and other issues with the report so we could trust the data it generated. Trusting the reliability of the data was critical for the fourth PDSA cycle.

PDSA cycle 4: tying outcomes to physician value-based compensation (July 2015-December 2015)

After developing a more robust reporting system, we decided to link compliance with LTVV to an already existing pay-for-performance programme. Although pay-for-performance programmes have shown inconsistent results with regard to improvement in outcomes, including studies in critical care medicine, the attending physicians in the Department of Critical Care Medicine were already participating in a health system-wide value-based compensation programme. ${ }^{20-22}$ Under this programme, three quality metrics were chosen by each department and division for every 6 months, and benchmarks were set for each metric. Quality metrics could be reused during the next measurement period if opportunity for improvement was still available. Staff other than physicians did not participate in this value-based compensation programme at UPMC. Compliance with LTVV $\leq 8 \mathrm{~mL} / \mathrm{kg}$ PBW was chosen as one of the quality 
metrics for the 6-month period of July 2015 to December 2015.

We used the revised EMR report to track compliance and report results from each ICU on a monthly basis beginning in July 2015. We provided the unblinded compliance report to all physicians in the department. The ICU medical directors played a large role in working with their local ICU physicians, directors of respiratory care and nursing leaders to audit and subsequently improve their performance in response to this report. When medical directors had questions about their data or had concerns regarding low compliance, our data management team was available and provided chart reviews of outlying patients.

\section{RESULTS}

Baseline data were obtained for the 1-year period previous to the first PDSA cycle (figure 1). Average compliance with LTVV was $55.7 \%$ at Presbyterian, $40.6 \%$ at Mercy and $60.1 \%$ at Magee. Data for Magee were available starting only in June 2012. In PDSA cycle 1, setting tidal volumes of $330 \mathrm{~mL}$ for women and $440 \mathrm{~mL}$ for men, compliance improved to $68.5 \%$ at Presbyterian, $68.5 \%$ at Mercy and $64.2 \%$ at Magee. PDSA cycle 2, education plus respiratory therapist-driven tidal volumes and implementation, proved to be the most effective PDSA cycle with an increase in LTVV compliance to $92.4 \%$ for Presbyterian, $85.8 \%$ for Mercy and $79.2 \%$ for Magee. PSDA cycle 3, modification of the EMR report, resulted in modest increases in compliance to $96.4 \%$ at Presbyterian, an unchanged compliance rate at Mercy and $81.7 \%$ at Magee. The physician value-based incentive in PDSA cycle 4 did not have a significant effect at Presbyterian with a compliance rate of $96 \%$, but did have a significant effect at Mercy with an increase to $95.8 \%$ as well as Magee with an increase to $91 \%$.

This quality initiative was highly sustainable with continued high rates of compliance with LTVV for years after the active PDSA cycles (figure 1).

LTVV compliance rates varied among the two control group care units. While the baseline rate of LTVV compliance in the Presbyterian MICU was higher than those of the ICUs that participated in the quality initiative at $78.8 \%$, it remained unchanged during and after the quality initiative in the Department of Critical Care Medicine ICUs (figure 2). Rates of compliance with LTVV in the Presbyterian coronary care unit (CCU) increased from $46.9 \%$ to $89.5 \%$ in the time period that the quality initiative was occurring in the other ICUs (figure 3).

There is a higher degree of variability in figures $1 \mathrm{C}$ and 3 because the magnitude of the CI is inversely related to the number of observations, in this case mechanically ventilated ICU patient days, aggregated into each run chart. For example, the Presbyterian data (figure 1A) are an aggregation of 100 beds across 6 ICUs. This displays the smallest variation. The Mercy Hospital data (figure 1B) consist of 3 ICUs with a total of 53 beds and have slightly wider CIs than the Presbyterian Hospital. The Magee Hospital data (figure 1C) are a single, 14-bed ICU with the widest CIs of all the intervention groups. The Presbyterian MICU (figure 2) in the control group is a 24-bed ICU while the Presbyterian Hospital CCU (figure 3) comprises only 10 beds and has the widest CIs. Data on ICU beds and annual admission are found in table 1 .

\section{Lessons and limitations}

It is important to remember that in this initiative LTVV was applied to all mechanically ventilated patients rather than limited to those with formally diagnosed ARDS. We used LTVV as what has been described as a default option, and using this default option for all mechanically ventilated patients has been shown to increase compliance with LTVV. ${ }^{23}{ }^{24}$ Default LTVV has helped bypass the well-established problem of poor clinical recognition of ARDS. ${ }^{25}$ Our intervention was also applied in the early steps of patient care-from the moment of admission to the ICU or intubation in the ICU—which also likely helped with the success of the initiative. Studies have shown that most ventilator changes occur in the first 12 hours of a ventilator episode and are often not changed thereafter. ${ }^{8}$ It has also been shown that initiation of LTVV as early as the patient presents to the hospital in the emergency department results in an increase in compliance with LTVV that extends beyond the emergency department and throughout their ICU course, despite no targeted intervention in the ICU. This further supports the finding that once initial ventilator settings are established, they are rarely changed. ${ }^{26}$

We also found benefits to having the respiratory therapist, not the physician, set the initial tidal volume. Although the cardiac ICU and the MICU at UPMC Presbyterian were not part of this QI initiative (table 1), we noted that the cardiac ICU had a significant improvement in LTVV compliance (figure 3) while the MICU remained virtually unchanged in their LTVV compliance rates throughout the initiative (figure 2). The 10 ICUs where the QI interventions were applied are all staffed by the Department of Critical Care Medicine. These include all three ICUs at Mercy Hospital, the one ICU at Magee Hospital and six of the eight ICUs at Presbyterian Hospital. These were chosen as the intervention ICUs because the QI project originated within the Department of Critical Care Medicine. Physician staffing in the two control ICUs at Presbyterian Hospital is through the Pulmonary Division and Cardiology Division of the Department of Medicine for the MICU and CCU, respectively. These ICUs did not participate in the QI initiative, however their data were collected and reported for comparison and use as a natural control group.

The respiratory therapists that staff the cardiac ICU (supervised by cardiology) also staff the neurological ICU (supervised by critical care medicine). The improvement in compliance with LTVV in the cardiac ICU control group suggests that the respiratory therapists who staff both the neurological and cardiac ICUs carried their new 
LTVV practice with them to the cardiac ICU, where they were responsible for setting the initial tidal volumes. The physician group in the cardiac ICU had the ability to set or change the tidal volumes at any time, yet it appears that this did not happen often. The results in the cardiac ICU likely reflect that the respiratory therapists were setting low tidal volumes and the physicians in that ICU did not feel the need to alter them (figure 3). This finding is consistent with prior studies that support the concept that placing the responsibility for complying with ventilator protocols, such as mechanical ventilator weaning protocols and spontaneous breathing trials, in the hands of respiratory therapists increases compliance with performance metrics. ${ }^{20} 27$

It must be noted that the MICU and cardiac ICU treat different patient populations. Respiratory failure requiring mechanical ventilation in the cardiac ICU is likely primarily due to pulmonary oedema while the types of respiratory failure requiring mechanical ventilation in the MICU is likely more heterogeneous, including a greater portion of patients with obstructive lung disease who may not have tolerated LTVV as well.

While there may be valid physiological rational for higher tidal volumes in the MICU at Presbyterian University Hospital (eg, a higher proportion of hypercarbic respiratory failure as compared with hypoxaemic respiratory failure), these findings suggest that providing respiratory therapy education and placing the responsibility of setting the initial tidal volume with the respiratory therapists were likely the most effective interventions that we deployed with our PDSA cycles. These would have been the only interventions that mechanically ventilated patients in the cardiac ICU would have been exposed to, while the patients in the MICU were exposed to none of the PDSA interventions and had minimal change in their rates of non-compliant ventilator days.

Although our rates of compliance are high and have endured for the years following the intervention, it is possible that spending more of our efforts on respiratory therapy education and compliance may have yielded even better results. The electronic report generated in PDSA cycle 3 was distributed only to physicians who may or may not have communicated it to their respiratory therapy colleagues. We likely missed an opportunity for further improvement by not providing this monthly report directly to respiratory therapists and their leadership as well.

We were limited in our data collection and analysis capabilities from our electronic health record. We were able to produce only an average tidal volume for each patient ventilator day rather than analyse the amount of time spent at each charted tidal volume. This may have masked some outlying high ventilator settings. As other research has noted, using a mean tidal volume does not provide a complete picture of LTVV compliance and does not correlate with hospital mortality at a patient level. ${ }^{8}$ Also, because the intervention was applied to all ventilated patients, we are unable to examine how LTVV compliance affected the subgroup with ARDS, which stands to benefit most from the intervention. Ideally, the subgroup of patients with ARDS should have a compliance with LTVV of near $100 \%$.

\section{CONCLUSION}

LTVV improves mortality in patients with ARDS and is associated with decreased morbidity and mortality in mechanically ventilated patients without ARDS. ${ }^{1215}$ Our QI project succeeded in improving compliance with LTVV in all ICUs that participated in the project. The improvement in LTVV compliance seen in the cardiac ICU control group indicates that this improvement was in large part due to education of respiratory therapists and an implementation strategy that gave them the responsibility of selecting the initial tidal volume. This conclusion is further supported by the largest increase in compliance being seen in PDSA cycle 2, which focused on education and a respiratory therapist-led intervention. Although the PDSA cycles of our project were from 2013 to 2015, our compliance with LTVV has been sustained, indicating that our QI project has resulted in a long-term change in practice.

Twitter Scott R Gunn @Scott_Gunn_

Acknowledgements The authors would like to thank Ms Kendra Williamson for her help with this manuscript.

Contributors CD and SRG contributed to all aspects of this work including development and implementation of the quality improvement project, data collection and analysis and writing of the manuscript. MC-M contributed to the data collection and analysis. ED contributed to the project development and implementation. CD is the guarantor of this work.

Funding The authors have not declared a specific grant for this research from any funding agency in the public, commercial or not-for-profit sectors.

Competing interests None declared.

Patient and public involvement Patients and/or the public were not involved in the design, or conduct, or reporting, or dissemination plans of this research.

Patient consent for publication Not required.

Ethics approval This project did not require institutional review board approval because it was a quality improvement project. This study involves human participants but did not require participation consent.

Provenance and peer review Not commissioned; externally peer reviewed.

Data availability statement Data are available upon request.

Open access This is an open access article distributed in accordance with the Creative Commons Attribution Non Commercial (CC BY-NC 4.0) license, which permits others to distribute, remix, adapt, build upon this work non-commercially, and license their derivative works on different terms, provided the original work is properly cited, appropriate credit is given, any changes made indicated, and the use is non-commercial. See: http://creativecommons.org/licenses/by-nc/4.0/.

\section{ORCID iDs}

Chenell Donadee http://orcid.org/0000-0002-9229-8507

Scott R Gunn http://orcid.org/0000-0002-6599-2018

\section{REFERENCES}

1 Acute Respiratory Distress Syndrome Network, Brower RG, Matthay MA, et al. Ventilation with lower tidal volumes as compared with traditional tidal volumes for acute lung injury and the acute respiratory distress syndrome. N Engl J Med 2000;342:1301-8. 
2 Futier E, Constantin J-M, Paugam-Burtz C, et al. A trial of intraoperative low-tidal-volume ventilation in abdominal surgery. $N$ Engl J Med Overseas Ed 2013;369:428-37.

3 From the American Association of Neurological Surgeons (AANS), American Society of Neuroradiology (ASNR), Cardiovascular and Interventional Radiology Society of Europe (CIRSE), Canadian Interventional Radiology Association (CIRA), Congress of Neurological Surgeons (CNS), European Society of Minimally Invasive Neurological Therapy (ESMINT), European Society of Neuroradiology (ESNR), European Stroke Organization (ESO), Society for Cardiovascular Angiography and Interventions (SCAI), Society of Interventional Radiology (SIR), Society of Neurolnterventional Surgery (SNIS), and World Stroke Organization (WSO), Sacks D, Baxter B, et al. Multisociety consensus quality improvement revised consensus statement for endovascular therapy of acute ischemic stroke. Int $J$ Stroke 2018;13:612-32.

4 Rhodes A, Evans LE, Alhazzani W, et al. Surviving sepsis campaign: international guidelines for management of sepsis and septic shock: 2016. Crit Care Med 2017;45:486-552.

5 Fan E, Del Sorbo L, Goligher EC, et al. An official American thoracic Society/European Society of intensive care Medicine/Society of critical care medicine clinical practice guideline: mechanical ventilation in adult patients with acute respiratory distress syndrome. Am J Respir Crit Care Med 2017;195:1253-63.

6 Papazian L, Aubron C, Brochard L, et al. Formal guidelines: management of acute respiratory distress syndrome. Ann Intensive Care 2019;9:69.

7 Bellani G, Laffey JG, Pham T, et al. Epidemiology, patterns of care, and mortality for patients with acute respiratory distress syndrome in intensive care units in 50 countries. JAMA 2016;315:788-800.

8 Sjoding MW, Gong MN, Haas CF, et al. Evaluating delivery of low tidal volume ventilation in six ICUs using electronic health record data. Crit Care Med 2019;47:56-61.

9 ARDS Definition Task Force, Ranieri VM, Rubenfeld GD, et al. Acute respiratory distress syndrome: the Berlin definition. JAMA 2012;307:2526-33.

10 Thompson BT, Chambers RC, Liu KD. Acute respiratory distress syndrome. N Engl J Med Overseas Ed 2017;377:562-72.

11 Rubenfeld GD, Caldwell E, Peabody E, et al. Incidence and outcomes of acute lung injury. N Engl J Med Overseas Ed 2005;353:1685-93.

12 Herridge MS, Tansey CM, Matté A, et al. Functional disability 5 years after acute respiratory distress syndrome. N Engl J Med 2011;364:1293-304.
13 Guérin C, Reignier J, Richard J-C, et al. Prone positioning in severe acute respiratory distress syndrome. $N$ Engl J Med Overseas Ed 2013;368:2159-68.

14 The National Heart, Lung, and Blood Institute PETAL Clinical Trials Network. Early neuromuscular blockade in the acute respiratory distress syndrome. N Engl J Med Overseas Ed 2019;380:1997-2008.

15 Serpa Neto A, Cardoso SO, Manetta JA, et al. Association between use of lung-protective ventilation with lower tidal volumes and clinical outcomes among patients without acute respiratory distress syndrome: a meta-analysis. JAMA 2012;308:1651-9.

16 Sutherasan Y, Vargas M, Pelosi P. Protective mechanical ventilation in the non-injured lung: review and meta-analysis. Crit Care 2014;18:211.

17 Weiss $\mathrm{CH}$, Baker DW, Weiner S, et al. Low tidal volume ventilation use in acute respiratory distress syndrome. Crit Care Med 2016;44:1515-22.

18 KnowWare International I. QIMacros. Denver, CO, 2020.

19 Fryar CD, Gu Q, Ogden CL. Anthropometric reference data for children and adults: United States, 2007-2010. Vital Health Stat 11 2012;11:1-48.

20 Barbash IJ, Pike F, Gunn SR, et al. Effects of Physician-targeted pay for performance on use of spontaneous breathing trials in mechanically ventilated patients. Am J Respir Crit Care Med 2017;196:56-63.

21 Glickman SW, Ou F-S, DeLong ER, et al. Pay for performance, quality of care, and outcomes in acute myocardial infarction. JAMA 2007;297:2373-80.

22 Kristensen SR, Meacock R, Turner AJ, et al. Long-Term effect of hospital pay for performance on mortality in England. $N$ Engl $\mathrm{J}$ Med Overseas Ed 2014;371:540-8.

23 Halpern SD, Ubel PA, Asch DA. Harnessing the power of default options to improve health care. N Engl J Med 2007;357:1340-4.

24 Bourdeaux CP, Thomas MJ, Gould TH, et al. Increasing compliance with low tidal volume ventilation in the ICU with two nudge-based interventions: evaluation through intervention time-series analyses. BMJ Open 2016;6:e010129.

25 Laffey JG, Pham T, Bellani G. Continued under-recognition of acute respiratory distress syndrome after the Berlin definition: what is the solution? Curr Opin Crit Care 2017;23:10-17.

26 Fuller BM, Ferguson IT, Mohr NM, et al. Lung-Protective Ventilation Initiated in the Emergency Department (LOV-ED): A QuasiExperimental, Before-After Trial. Ann Emerg Med 2017;70:406-18.

27 Ely EW, Baker AM, Dunagan DP, et al. Effect on the duration of mechanical ventilation of identifying patients capable of breathing spontaneously. N Engl J Med 1996;335:1864-9. 